\title{
Growth of ultrathin cobalt oxide films on $\operatorname{Pt}(111)$
}

\author{
Maurizio De Santis, ${ }^{1,2,}$ Andreas Buchsbaum, ${ }^{1}$ Peter Varga ${ }^{1}$ and Michael Schmid, ${ }^{1}$ \\ ${ }^{1}$ Institut für Allgemeine Physics, Technische Universität Wien, A-1040 Wien, Austria. \\ ${ }^{2}$ Institut Néel, CNRS and UJF, BP 166, F-38042 Grenoble Cedex 9, France
}

Cobalt surface oxides where grown on $\operatorname{Pt}(111)$ by depositing Co and dosing with molecular oxygen at temperatures ranging between $300 \mathrm{~K}$ and $740 \mathrm{~K}$. Oxidation of 1 monolayer (ML) Co results in a two-dimensional (2D) moiré structure, observed both using low energy electron diffraction and scanning tunneling microscopy, and interpreted as a polar (oxygen terminated) $\mathrm{CoO}(111)$ atomic bilayer. With respect to bulk $\mathrm{CoO}$, it is expanded by $2.7 \pm 0.5 \%$ in the surface plane. An almost flawless moiré pattern is obtained after a final step of annealing at $740 \mathrm{~K}$ in oxygen. Insufficient oxidation leads to defects in the moiré pattern, consisting of triangular dislocation loops of different sizes; the smaller ones occupying one half of the moiré cell. Low-temperature annealing (450 K) can be used to create a zigzag phase, which is mainly observed in 1-ML thick areas after several cycles of Co deposition (1 ML each) and oxidation at $10^{-7}$ mbar. The $\mathrm{CoO}$ films obtained by deposition/oxidation cycles exhibit Stranski-Krastanov growth; the structure of the 2D layer in between the islands depending on the thermal treatment. After annealing at $740 \mathrm{~K}$ it exhibits the moiré pattern, while the zigzag phase was observed after low-temperature annealing. The second monolayer consists of a moiré pattern different from that of the 1st layer, presumably a wurtzite-like structure. Above the 3rd layer, we observe only small 3D islands, which exhibit a band gap. We have also studied oxidation of surface alloys obtained by depositing Co and annealing. On these surfaces, we found a quasi- $(3 \times 3)$ reconstruction. Structure models are presented for all phases observed, and we argue that some of the moiré-like structures might be useful as templates for metal cluster growth.

PACS numbers

68.47.Gh Oxide surfaces

68.37.Ef Scanning tunneling microscopy (including chemistry induced with STM)

68.55.J- Morphology of films

68.55.A- Nucleation and growth 


\section{INTRODUCTION}

Ultrathin transition metal oxide (TMO) films are investigated mainly for their catalytic ${ }^{1,2}$ and magnetic ${ }^{3}$ properties. Concerning the latter the most important domain of application is the exploitation of exchange anisotropy or exchange bias occurring at the interface between antiferromagnetic oxides and ferromagnets. ${ }^{4}$ Such exchange interaction systems are employed in spin valves and in systems showing giant magnetoresistence. A further interesting effect of exchange bias is to overcome the superparamagnetic limit in nanostructures, as it happens for Co nanoparticles embedded in a $\mathrm{CoO}$ matrix. ${ }^{5}$ In all these applications, a key parameter for enhancing the exchange interaction is the control of the interface structure at the atomic level, which determines the strength of the exchange coupling. ${ }^{3}$

$\mathrm{CoO} / \mathrm{Co}$ bilayers reveal an extremely strong exchange bias ${ }^{6}$ and are interesting both for fundamental studies and applications. Bulk $\mathrm{CoO}$ is antiferromagnetic with a Néel temperature $T_{N}$ of $293 \mathrm{~K}$ and a large magnetic anisotropy. ${ }^{3}$ Larger Néel temperatures were obtained in rather thick $\mathrm{CoO} / \mathrm{NiO}$ superlattices, ${ }^{7}$ at the price of a reduction of the anisotropy. A higher Néel temperature than $\mathrm{CoO}$ was also observed for shorter-period superlattices, $3 \mathrm{ML} \mathrm{CoO}+3 \mathrm{ML} \mathrm{NiO}$, coupled to an ultrathin ferromagnetic $\mathrm{PtCo}(111)$ layer, as evidenced by a the observation of exchange bias up to $\approx 320 \mathrm{~K}$ in this system. ${ }^{8}$ Above this temperature, the interfacial $\mathrm{CoO}$ is polarized by the PtCo layer and contributes to the total ferromagnetic signal. Again, the knowledge of the structure of the buried interface is fundamental in the understanding of such phenomena.

Similar to alumina films, ${ }^{9,10}$ the large supercells of epitaxial ultrathin TMO films grown on metals are also used as templates for self-assembling arrays of metal nanoclusters, as shown in the cases of the moiré or zigzag pattern resulting from the growth of a single $\mathrm{FeO}$ bilayer on $\mathrm{Pt}(111)$ ${ }^{11,12}$ and $\mathrm{TiO}_{\mathrm{x}} / \mathrm{Pt}(111),{ }^{13}$ as well as the $(4 \times 4)$ vanadium oxide mesh on $\mathrm{Pd}(111) .{ }^{14}$ Also these applications require understanding the surface structure of the oxide films.

At room temperature, $\mathrm{CoO}$ has the rocksalt crystal structure, with lattice constant $a_{\mathrm{CoO}}=$ 425.4 pm (Ref. 7). Each (111) plane contains one kind of atoms only, alternatively Co and O, disposed in an hexagonal mesh with the in-plane interatomic distance of $301 \mathrm{pm}$. Below $T_{N}$ it orders with parallel spins on (111) planes, and with anti-parallel spin directions on adjacent (metal) planes. The $\mathrm{CoO}(111)$ surface thus exhibits the largest density of uncompensated spins and hence a priori the largest exchange interaction. Unfortunately this surface is polar, thus the calculated (111) surface energy for the bulk material is infinite. ${ }^{15,16}$ This divergence can be suppressed by various mechanisms, ${ }^{17}$ e.g., surface reconstructions, ${ }^{18}$ a crystallographic structure at the surface different from that in the bulk, ${ }^{19}$ vacancy islands, ${ }^{20}$ reduced charge of the surface ions, ${ }^{21}$ non-stoichiometric 
surfaces $^{22}$ or adsorbates. ${ }^{23}$ For ultrathin films, the polar surface problem is alleviated, nevertheless it reduces the stability of the films, making it difficult to achieve layer-by-layer growth of wellordered $\mathrm{CoO}(111)$ films.

The structure of ultrathin epitaxial $\mathrm{CoO}$ films on several substrates, like for example $\operatorname{Ir}(100)$ (Ref. 24) and $\operatorname{Pd}(100)$ (Ref. 25), was already studied mainly by scanning tunneling microscopy (STM) and low energy electron diffraction (LEED). The growth of cobalt oxide on $\operatorname{Pt}(111)$ at room temperature (RT) was investigated employing reflection high-energy electron diffraction (RHEED)

and spectroscopic techniques. ${ }^{26}$ It was reported that cycles of alternate Co deposition (1 ML) and $\mathrm{O}_{2}$ dosing (300 Langmuir) at RT results in a non-reconstructed $\mathrm{CoO}(111)$ film, described as rather flat up to $6 \mathrm{ML}$. Our STM results are more sensitive to the roughness, which indeed is observed already at the beginning of the growth.

Here we present a detailed study in a large temperature range of the first growing stages of this oxide, combining STM, LEED and Auger electron spectroscopy (AES). Several surface cobalt oxide phases were found, depending on the preparation.

\section{EXPERIMENTAL AND METHODS}

The $\operatorname{Pt}(111)$ substrate was prepared by cycles of $2 \mathrm{keV} \mathrm{Ar}^{+}$sputtering and annealing at about $1070 \mathrm{~K}$ in an ultrahigh vacuum (UHV) chamber with a base pressure in the low $10^{-10}$ mbar range. At this stage no impurities could be detected by AES. Co was deposited from a pure rod using a liquid-nitrogen-cooled electron beam evaporator. During deposition a retarding voltage was applied to the end of the evaporator to suppress high-energy metal ions, which could modify the growth. ${ }^{27}$ The deposition rate, calibrated with a quartz crystal microbalance, was typically $1 / 3$ monolayer (ML) per minute [we define $1 \mathrm{ML}$ as the number of atoms in a $\operatorname{Pt}(111)$ monolayer]. Then, the film was annealed without oxygen at different temperatures (leading to alloying at high $T$ ). Unless specified otherwise, the oxide was formed by dosing molecular oxygen at about $10^{-7}$ mbar for 10 minutes, resulting in an exposure to 50 \pm 10 Langmuir (L; 1 Langmuir corresponds to a dose of $10^{-6}$ torr $\times s$ ). A large interval of the oxidation temperature was spanned during the experiments, from room temperature (RT) to $740 \mathrm{~K}$. STM, LEED, AES and LEIS (low energy ion scattering) measurements were performed in a connected UHV chamber with a base pressure below $5 \times 10^{-11}$ mbar. The STM measurements were performed using a customized commercial STM (Omicron $\mu-$ STM) with an electrochemically etched W-tip. All STM images were obtained in constant current mode with the voltage bias $\left(V_{s}\right)$ applied to the sample. Lattice constants of moiré structures were determined by selecting the spots corresponding to either the substrate or the overlayer in the 
Fourier transform of STM images and doing a backtransform, then measuring the lattice constant in the same area of the image. As this procedure eliminates any differences of scanner calibration between different images as well as the distortions within an image, it allows us to reach an accuracy of about $0.5 \%$. AES spectra were collected with a cylindrical mirror analyzer with a concentric electron gun. LEIS was measured with $1 \mathrm{keV} \mathrm{He}^{+}$ions at a scattering angle of $90^{\circ}$.

\section{RESULTS AND DISCUSSION}

\section{A. The moiré structure.}

As a first step we studied the exposure to oxygen of $1 \mathrm{ML} \mathrm{Co/Pt(111)} \mathrm{as} \mathrm{function} \mathrm{of} \mathrm{the}$ temperature. Fig. 1(a) shows an STM image of about $1 \mathrm{ML}$ of Co deposited at RT on the substrate, and then dosed with $50 \mathrm{~L} \mathrm{O}_{2}$ at RT (without any annealing of the as-deposited Co film). It is known that previously to the oxidation this surface is covered by large islands of monolayer thickness coexisting with uncovered Pt regions and with areas showing a second Co layer on top of the first one. ${ }^{28}$ In the figure we observe small clusters, about $250 \mathrm{pm}$ in height and $2 \mathrm{~nm}$ in size, resulting from the oxidation of previously Co covered areas, while no islands appear on Pt areas (dark regions). We explain the presence of clusters with the expulsion of atoms from the underlying Co layer during oxidation: a bulk $\mathrm{CoO}(111)$ plane has a $\mathrm{Co}$ atomic density $15 \%$ lower than pseudomorphic $\mathrm{Co} / \mathrm{Pt}(111)$; the difference is actually larger due a slight contraction $^{28}$ of the asdeposited Co on the $\operatorname{Pt}(111)$ surface. The large dendrites formed when depositing Co submonolayers on Pt(111) (Ref. 29) are also observed on the sample shown in Fig.1(a) after oxidation. These dendrites should consist mainly of $\mathrm{Pt}^{29}$ and almost no clusters form on them. 

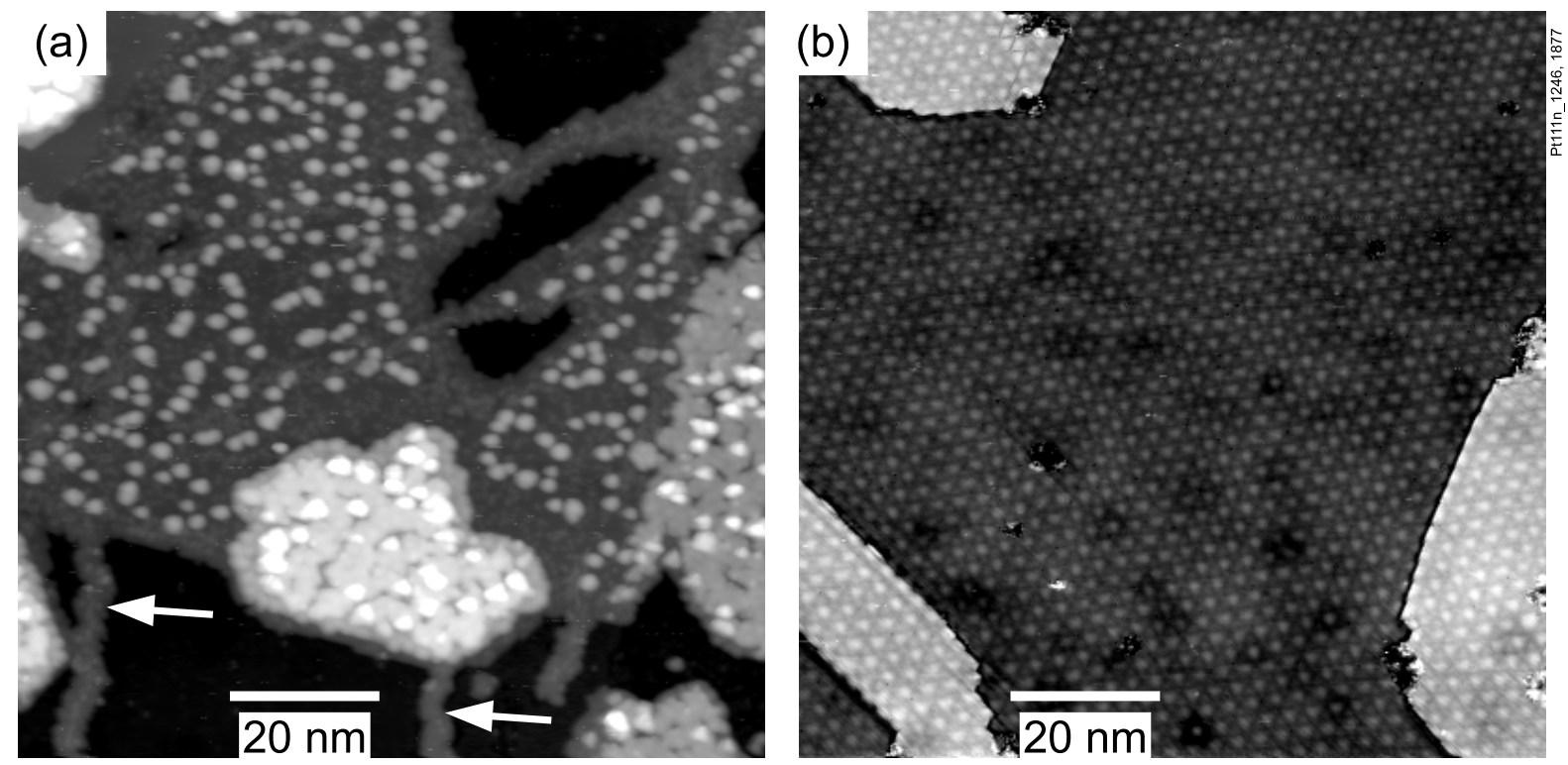

FIG. 1. STM images $\left(V_{s}=+0.5 \mathrm{~V}, I_{t}=0.1 \mathrm{nA}\right)$ of $1 \mathrm{ML} \mathrm{Co} / \mathrm{Pt}(111)$ deposited at RT and (a) dosed with $50 \mathrm{~L} \mathrm{O}$, (b) annealed and dosed with $\mathrm{O}_{2}$ at $570 \mathrm{~K}$, then annealed under oxygen at $740 \mathrm{~K}$. The arrows in (a) indicate two dendrites. Image processing has been used in (b) to reduce the step height, making the corrugation on the terraces more apparent.

An almost flawless and flat surface oxide [Fig. 1(b)] was obtained by using two different annealing temperatures: $1 \mathrm{ML}$ of Co was deposited at RT and annealed at $570 \mathrm{~K}$, to get a flat Co atomic layer partly alloyed with $\mathrm{Pt}$; then at this same temperature $\mathrm{O}_{2}$ was dosed (dose: $50 \mathrm{~L}$ ); and finally it was annealed for 10 minutes at $740 \mathrm{~K}$ in $10^{-8} \mathrm{mbar}_{2}$. The first oxidation step is performed at a temperature low enough to avoid Co diffusion into the near-surface Pt layers before an oxide forms. The second step then results in an oxide layer with a very low defect concentration. The large scale STM of Fig. 1(b) shows a moiré pattern, which extends over the image size (100 $\mathrm{nm}$ ) with a very low density of defects. The moiré is observed on all the terraces, and its period is $\Lambda$ $=2.70 \pm 0.05 \mathrm{~nm}$. The interatomic distance $d_{\text {oxide }}=309 \pm 2 \mathrm{pm}$, obtained by atomically resolved images, is expanded by about $11 \%$ with respect to the Pt substrate (interatomic distance $d_{\mathrm{Pt}}=277.5$ $\mathrm{pm})$. These values satisfy the moiré equation for an unrotated overlayer: $1 / \Lambda=1 / d_{P t}-1 / d_{\text {oxide }}=(2.7$ $\pm 0.1 \mathrm{~nm})^{-1}$. The same misfit is observed in the LEED pattern (Fig. 2), which is homogeneous on the entire surface. Compared to bulk $\mathrm{CoO}(111)$, the in-plane distances are expanded by $2.7 \pm 0.6 \%$. LEIS on this same surface shows contributions mainly from $\mathrm{Co}$ and $\mathrm{O}$ atoms, the Pt peak being only a few percent of the total signal. Assuming that the Pt signal comes from uncovered Pt areas, comparison with standard spectra of pure Pt indicates that $\leq 1 \%$ of the surface is pure Pt. Concerning the stoichiometry of the films, the STM images do not allow us to determine whether we have a $\mathrm{CoO}$ bilayer like $\mathrm{FeO} / \mathrm{Pt}(111)$ (Refs. 30, 31, 32 and 33) or a O-Co-O trilayer structure like the O-Rh-O surface oxide obtained by oxidation of Rh(111) (Ref. 34). The latter can be 
excluded by AES, by comparison with the film oxidized at room temperature (where formation of a trilayer oxide is unlikely; the perfect moiré has only a few percent higher oxygen content than the RT-oxidized film) and also by comparison of AES data with those of the trilayer oxide on $\mathrm{Rh}(111)$.

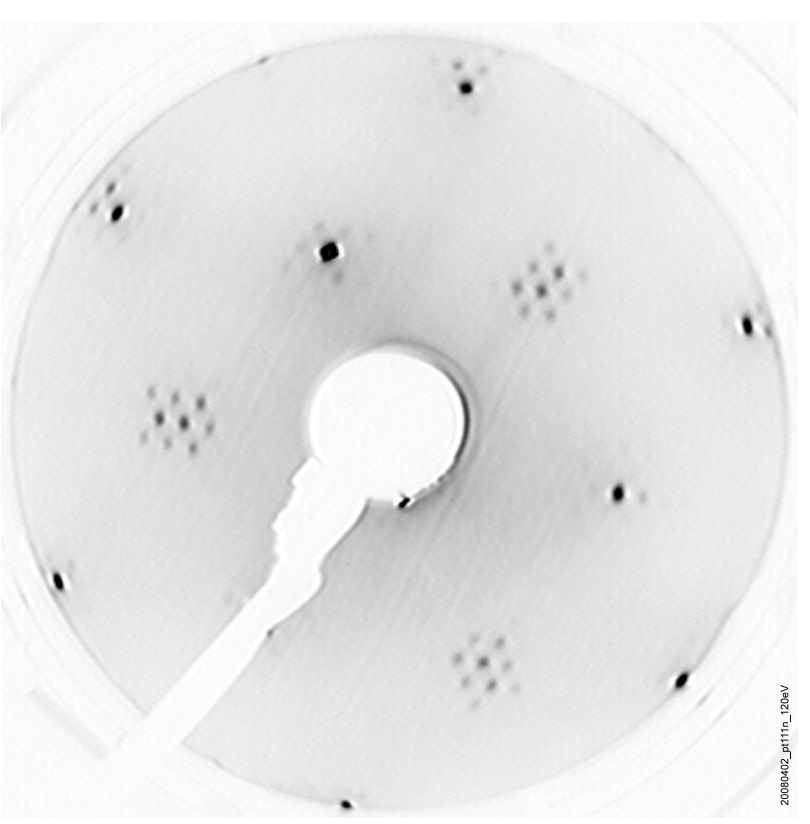

FIG. 2. LEED pattern of the moiré shown in Fig. 1(b) $(E=120 \mathrm{eV})$.

Thus, this structure consists of a single $\mathrm{CoO}$ bilayer, $\mathrm{O}$ terminated and slightly in-plane expanded. Beside the analogy with the $\mathrm{FeO}$ case, such a kind of termination is the only one that allows explaining coherently the oxygen deficient structures discussed in the next paragraphs. The expansion is similar to the value of $2 \%$ found for $\mathrm{FeO} / \mathrm{Pt}(111) \cdot{ }^{30,32}$ For the $\mathrm{FeO}$ films this expansion was accompanied by a strong contraction of the interlayer spacing (68 pm compared to $125 \mathrm{pm}$ in bulk $^{33}$ ). Such a reduction of buckling is likely to occur also in our $\mathrm{CoO}$ films, decreasing the dipole moment and, hence, the electrostatic energy. At variance with the iron oxide case, the moiré pattern that we observe is not rotated compared to the Pt(111) surface mesh. The need for a two-step annealing process for the $\mathrm{CoO}$ film, not required for $\mathrm{FeO}$, might be related to the higher solubility of Co in Pt as compared to Fe. ${ }^{35}$ 

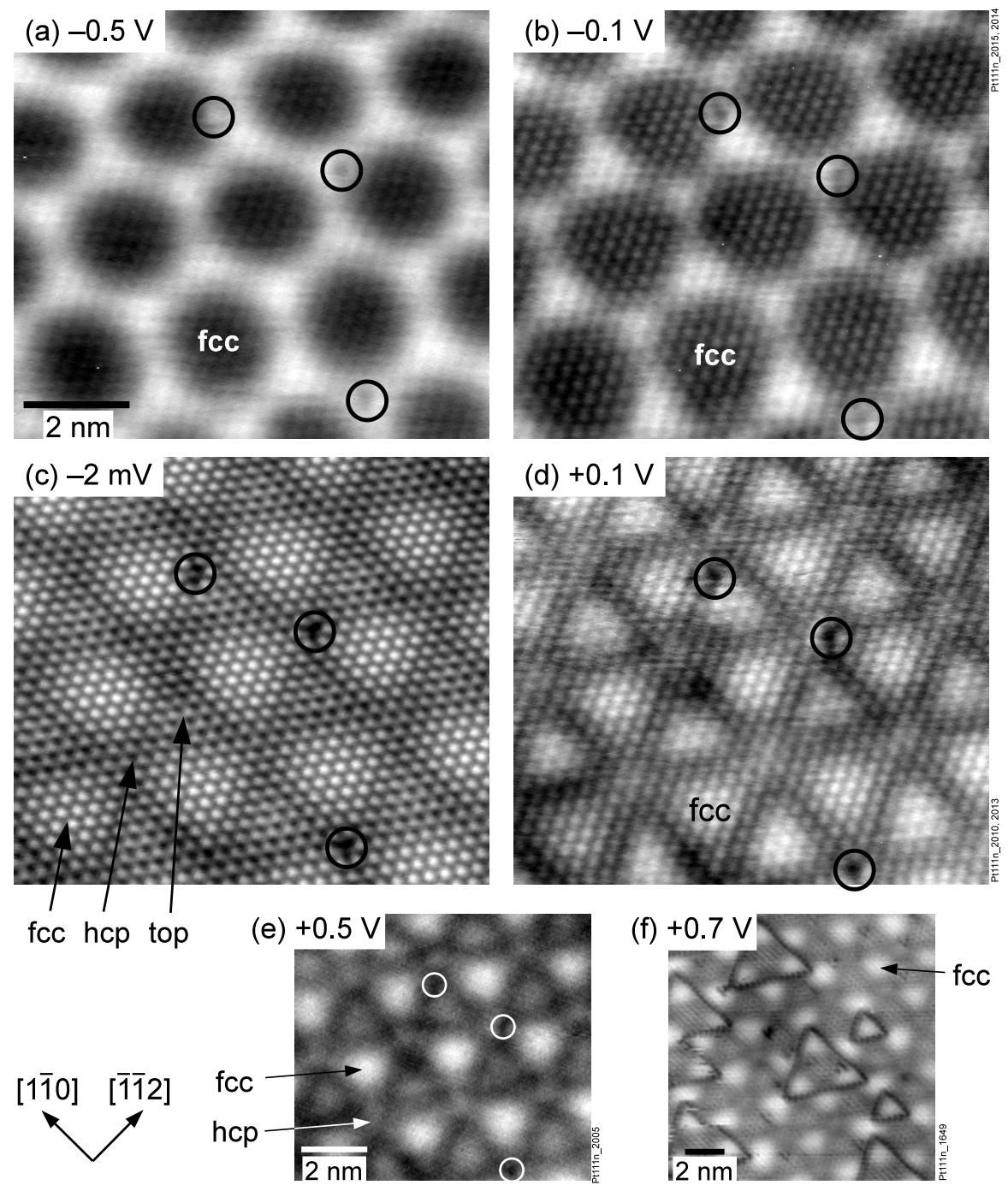

FIG. 3. STM images of the $\mathrm{CoO} / \mathrm{Pt}(111)$ moire structure. In (a)-(e) the same region is imaged with different sample bias ( $I_{t}=1 \mathrm{nA}$ for all). Regions with Co in fcc, hcp and on-top sites of the Pt substrate atoms are identified by comparison with images of the triangular stacking faults. Images (a)-(e) were obtained on the 2D layer of a sample obtained with 3 cycles of $1 \mathrm{ML}$ Co deposition and $\mathrm{O}_{2}$ exposure at $570 \mathrm{~K}$, followed by annealing at $740 \mathrm{~K}$ in $1 \times 10^{-8}$ mbar $\mathrm{O}_{2}$ (see section III.D); circles mark the same 3 defects in (a)-(e). Image (f) shows dislocation triangles (1 ML Co, 50 L $\mathrm{O} 2$ at $620 \mathrm{~K}$, then annealed at $\left.740 \mathrm{~K} ; I_{\mathrm{t}}=0.1 \mathrm{nA}\right)$.

Fig. 3(a-e) shows the moiré imaged with different bias. The registry of the images can be inferred from a few point defects (circles). Depending on the sample bias voltage, the appearance of the moiré pattern changes, which evidences the role of surface electronic states in the corrugation observed by STM. It is in particular clear that the honeycomb feature in Fig. $3(a, b)$ is not related to the geometric corrugation of the moiré structure, because the atomic corrugation is strongly reduced in the bright honeycomb regions, where it seems that a smeared-out electronic state dominates tunneling. Voltage-dependent appearance of the oxide has been observed previously also in the $\mathrm{FeO} / \mathrm{Pt}(111)$ case, but less pronounced as there the atomic corrugation seemed to be not 
significantly reduced in areas of high density of states. ${ }^{36}$ [It should be noted that the images shown in Fig. 3(a-e) were obtained on a sample with 3 cycles of Co deposition (1 ML each) and annealing in oxygen at $570 \mathrm{~K}$, imaging the first-layer moiré pattern between the $3 \mathrm{D}$ islands. The images of the first-layer moiré are the same for one or several deposition cycles, and also the in-plane lattice constant is the same, $309 \mathrm{pm}]$.

When comparing voltage-dependent images of this moiré structure with those of the dislocation triangles (see next section), we can determine the stacking of the different areas in the moiré. The areas with the atoms appearing as well-defined protrusions at very low tunneling voltages [Fig. 3(c), Fig. 4(a)] will be identified as those with fcc stacking; these areas appear dark at negative sample voltages $V_{\mathrm{s}}$ and bright at $V_{\mathrm{s}} \geq 0.1 \mathrm{~V}$.

\section{B. Dislocation triangles in the moiré structure.}

Preparation with insufficient oxidation results in a moiré pattern with a large density triangular dislocation loops. The STM image of Fig. 4(a) was measured after deposition of 1 ML of Co on $\operatorname{Pt}(111)$ at RT, followed by annealing $(10 \mathrm{~min}$. at $640 \mathrm{~K})$ and $\operatorname{dosing} \mathrm{O}_{2}(\approx 50 \mathrm{~L})$ at a temperature of $640 \mathrm{~K}$, but without the post-annealing in $\mathrm{O}_{2}$ required to produce the perfect moiré structure. Within the dislocation loops, atoms at the surface follow a different stacking, as shown by the line of the arrow indicating the $\left[\begin{array}{lll}1 & \overline{1} & 0\end{array}\right]$ direction.

Fig. 4(b) shows a ball model of the moiré structure, with the periodicity approximated to the closest commensurate value, $\Lambda=10 d_{\mathrm{Pt}}(2.775 \mathrm{~nm})$. Co and $\mathrm{O}$ atoms follow the $\mathrm{Pt}(111)$ fcc stacking sequence $A B C$ in the lower right of the moiré cell shown in Fig. 4(b). Due to the misfit, the stacking switches to hcp in the upper right of the cell, while Co is on top of Pt at the cell corners. The twinned domain ( $A C B$ stacking in the registry position) would be obtained exchanging the in plane coordinates of $\mathrm{Co}$ and $\mathrm{O}$. On the perfect moiré surface, this twinned domain, if existing at all, cannot be present in a significant fraction of the surface, as evidenced by the 3-fold symmetry of the moiré spots in the LEED pattern (Fig. 2). Two domains with similar area would result in 6-fold symmetry of the LEED pattern (except for the substrate spots). Moreover, in the well-annealed moiré structure, no domain walls are observed by STM, also indicating that there is only one domain.

Merte, Knudsen and coworkers in the Århus group have studied similar dislocation triangles on

$\mathrm{FeO} / \mathrm{Pt}(111){ }^{37,38}$ It must be noted, however, that they have created the dislocation loops by chemically reducing the perfect $\mathrm{FeO}$ film with atomic hydrogen, while the structures observed by us are formed during growth. Not surprisingly considering the way of creating these defects, Merte et al. have interpreted their dislocations as defects of the oxygen layer only, with the Fe layer below 
remaining the same as in the unperturbed moire structure. Their model for the small triangles visible in Fig. 4(a) is shown in Fig. 4(c); it perfectly fits our STM images under the assumption that STM images the $\mathrm{O}$ atoms as calculated for FeO in Ref. 31 (in contrast to Ref. 37, we have never observed a tip change where the tip switches to imaging the other atom type as protrusions). Note the line in $\left[\begin{array}{ll}1 & \overline{1}\end{array}\right]$ direction, clearly indicating a different stacking inside the triangle. For $\mathrm{FeO}$, the driving force behind the formation of these defects was explained as a preference for fcc-like staking in the upper layers (Pt-Fe-O), avoiding the regions of hcp-like stacking in the unperturbed moiré. ${ }^{38}$

In our case, with the structures formed by annealing a Co film in oxygen, we also have to consider the possibility of a dislocation loop in the Co layer, enclosing an area with a stacking fault between the Pt and Co layers. Such a rearrangement would be clearly favorable if it could avoid the on-top sites of Co on Pt, which may be energetically even less favorable than hcp-like stacking. ${ }^{37}$ In the following, we will describe the two possible arrangements for triangular dislocation loops avoiding on-top sites. These structure models require a stacking fault between the Pt and Co layers. We will show that these models do not explain the STM image in Fig. 4(a), but one of these arrangements will be the basis for understanding the 'zigzag' phase in the following section.
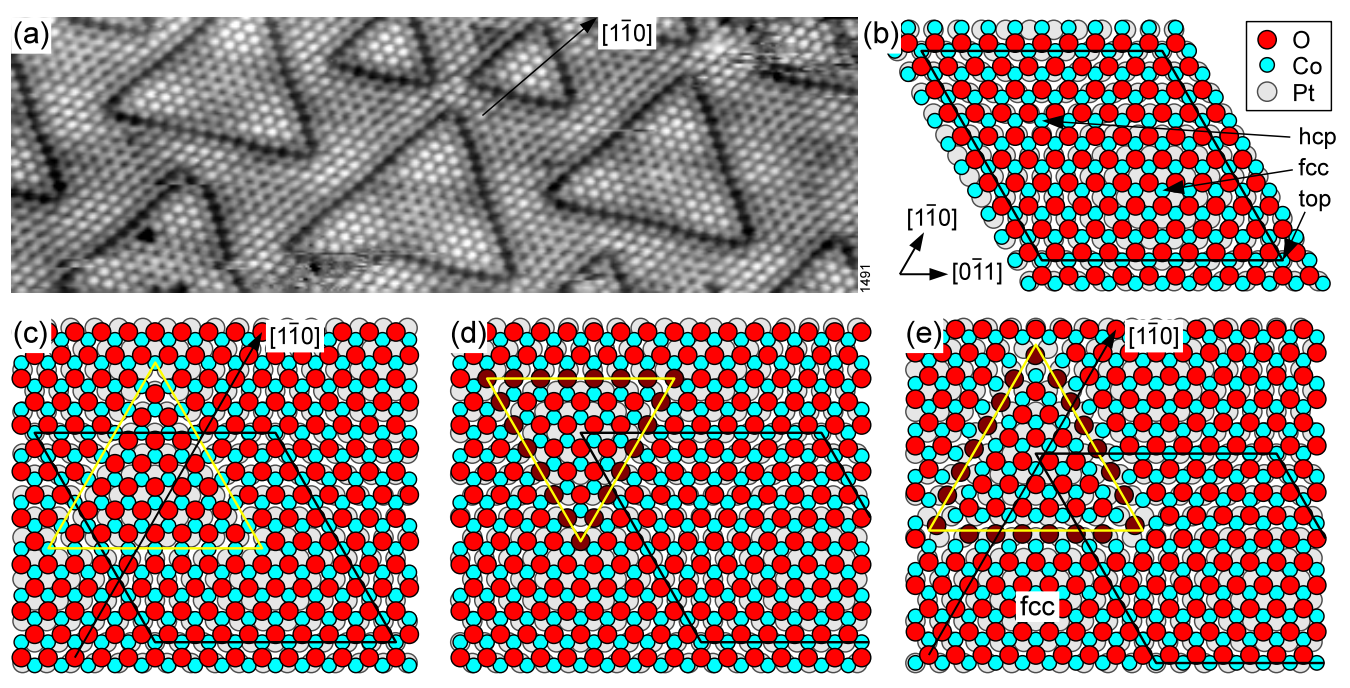

FIG. 4. (Color online) (a) $18 \times 6 \mathrm{~nm}^{2} \mathrm{STM}$ image of $1 \mathrm{ML} \mathrm{Co} / \mathrm{Pt}(111)$ annealed and exposed to $\mathrm{O}_{2}$ at $640 \mathrm{~K}\left(V_{\mathrm{s}}=-1 \mathrm{mV}\right.$, $I_{\mathrm{t}}=2 \mathrm{nA}$ ). (b)-(e) Ball models of the unperturbed moiré structure, and different types of triangular dislocation loops; we argue that (c) is the correct model. Oxygen atoms at the dislocations that are in asymmetric twofold or fourfold hollow sites are shown as dark (brown) filled circles.

The first of these models is shown in Fig. 4(d) (for simplicity, we consider only the small triangles here, with side lengths of $\approx 7$ atoms). In this case, the dislocation loop would be situated only in the Co layer, while the oxygen lattice could be uninterrupted. This model would require STM to show the Co atoms as protrusions. For obtaining a reasonable atomic arrangement at the triangle boundary, we have to invert the orientation of the triangle with respect to the one observed 
experimentally. This can only happen if the majority of the film does not grow with the same fcc stacking sequence as the Pt substrate, but with twinned stacking. This must be considered unlikely, however, because the stacking fault energy of $\mathrm{Co} / \mathrm{Pt}(111)$ must to be rather high, as evidenced by significantly different areas of fcc and hcp Co in the first monolayer; ${ }^{28}$ the same is expected to hold for the oxide. ${ }^{38}$

Assuming that the majority of the moiré is not twinned, we can create a model avoiding the on-top sites as shown in Fig. 4(e). The oxygen atoms at the dislocations [dark in Fig. 4(e)] are in asymmetric fourfold hollow sites, thus they would almost certainly appear different from those in the usual threefold sites, but this is not observed (this argument would be invalid assuming that STM images show Co, however). We will later see that this kind of dislocations does exist in a different structure, the 'zigzag' phase, and that STM indeed images the oxygen atoms. We can also exclude the model in Fig. 4(e) for another reason: The stacking sequence is the same (fcc) near the center of the triangles and immediately outside the triangle sides [marked ' $f c c$ ' in Fig. 4(e)]. The STM images show a different appearance at these positions, which strongly indicates different stacking. This is true for very low tunneling voltages [Fig. 4(a)] as well as for higher voltages, where electronic effects dominate [Fig. 3(f)]. Thus, there is no viable model for the triangular dislocation loops avoiding on-top Co, which means that the model in Fig. 4(c), not avoiding the ontop sites, must be the correct one.

As mentioned above, annealing in oxygen at $740 \mathrm{~K}$ removes the dislocation triangles, resulting in a well-ordered moiré. At the same time the ratio of the AES $\mathrm{O}(513 \mathrm{eV}) / \mathrm{Co}(778 \mathrm{eV})$ peak-to-peak heights increases by about $10 \%$. This is explained by the increase of the oxygen coverage when the oxygen-deficient dislocation loops are healed. Furthermore, at this temperature excess Co can diffuse to deeper Pt layers, below the probing depth of AES.

\section{The zigzag phase.}

The structure of the 2D layer obtained by oxidation at an even lower temperature of $450 \mathrm{~K}$ is quite different from the moiré. At this temperature, it is impossible to obtain sufficiently large 2D islands by depositing just 1 ML Co and annealing, thus we have obtained the structure by two cycles of Co deposition (1 ML each) and $\mathrm{O}_{2}$ dosing $(50 \mathrm{~L}$ each). The first ML was deposited at RT, then the sample was annealed at $450 \mathrm{~K}$; oxidization and the second deposition-oxidation cycle were done at $450 \mathrm{~K}$ as well. Similar to the moiré structure with a large number of dislocation triangles, AES indicates an $\mathrm{O} / \mathrm{Co}$ ratio about $10 \%$ below that of the perfect moiré. An atomically resolved STM image of the phase grown at $450 \mathrm{~K}$, obtained by zooming in a flat region between the 2-ML and 
higher islands, is shown in Fig. 5(a). The surface appears nanostructured with parallel dark rows, spaced by about $2.9 \mathrm{~nm}$. Inside the bands delimited by these rows the atoms are arranged in triangles, whose boundaries form a dark zigzag pattern.
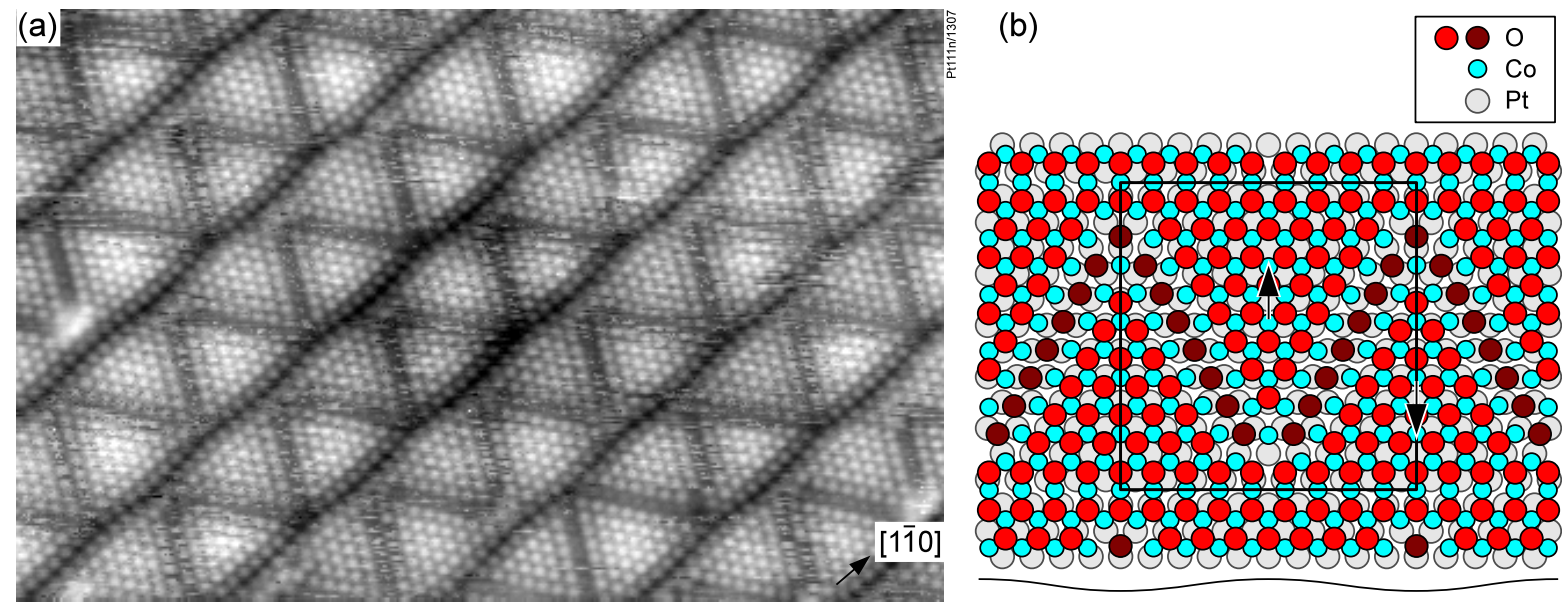

FIG. 5. (Color online) (a) STM image $\left(28 \times 12 \mathrm{~nm}^{2}, V_{s}=10 \mathrm{mV}, I_{t}=1 \mathrm{nA}\right)$ of the zigzag structure, obtained in a region between the islands after two cycles of Co deposition (1 ML) and $\mathrm{O}_{2}$ dosing $(50 \mathrm{~L})$ at $450 \mathrm{~K}$. (b) Ball model of the structure. Oxygen atoms at the zigzag dislocation line (shown darker) are in asymmetric fourfold hollow sites.

The structure is observed with slightly different cell sizes; the most frequent unit cell is a rectangular $(10 \times 6 \sqrt{3})$-rect cell $\left(2,775 \times 2,884 \mathrm{~nm}^{2}\right)$. Assuming that STM again shows the oxygen atoms as protrusions, and using the same building blocks as discussed above, we can derive a model of the structure: The appearance of the long dark lines along the $\left[\begin{array}{lll}1 & \overline{1} & 0\end{array}\right]$ direction in the STM image is similar to that of the dislocation triangles discussed above, with a locally rectangular arrangement of the $\mathrm{O}$ atoms. On the other hand, the hexagonal $\mathrm{O}$ lattice is only weakly disturbed at the dark zigzag lines, with one $\mathrm{O}$ row appearing darker, as one would expect for the structure in Fig. 4(e). The resulting structure model is shown in Fig. 5(b). This structure avoids the unfavorable on-top sites of Co that would occur in the unperturbed moiré structure. The zig-zag shaped line defects in the Co layer separate triangles with different orientations and different stacking, fcc and twinned fcc with respect to the substrate. The oxygen atoms at these line defects not only appear with different contrast (darker) as expected for their 4-fold sites in contrast to the 3-fold sites elsewhere, in many places they also appear frizzy, indicating that the line defect jumps back and forth one atomic row while the image is scanned. In dislocation terminology, the line defects in the Co layer are Shockley partial dislocations, which can easily glide in the (111) layer.

In the model shown in Fig. 5(b), the Co and $\mathrm{O}$ lattices have been modified by introducing the line defects only, further distortions have not been applied. In this figure, the areas of perfect fcc 
stacking are not the centers of the triangles but closer to the base (the straight line defect in the $\mathrm{O}$ layer). The STM images of the triangular dislocation loops [Fig. 4(a)] show the highest contrast of the $\mathrm{O}$ atoms in regions of perfect fcc stacking (the center of the triangles), and transferring this knowledge to the zigzag structure means that also here the perfect fcc stacking is actually observed closer to the center of the triangles. Thus, the $\mathrm{Co}$ and $\mathrm{O}$ atoms are slightly displaced in the direction of the arrows in Fig. 5(b). This is advantageous especially for the atoms near the tip of the triangles, where Fig. 5(b) shows that the Pt-Co-O stacking is close to the unfavorable hcp stacking. There, shifting the $\mathrm{CoO}$ overlayer in the direction of the arrows makes the stacking more fcc-like. As a result of this displacement, the atomic rows become slightly wiggly, as indicated by a wavy line at the bottom of Fig. 5(b). This is also observed in the STM image, where the diagonal dark defects in the $\mathrm{O}$ layer are not perfectly straight but slightly wiggly. The amplitude of these wiggles as estimated from the STM image is about $30 \mathrm{pm}$. The fcc stacking in the triangles is also confirmed by STM images at larger positive voltages (not shown), where the centers of the triangles appear brightest, similar to the fcc regions in Fig. 3(e,f).

The stoichiometry of the zig-zag unit cell shown in Fig. 5(b) is $\mathrm{Co}_{90} \mathrm{O}_{82}$, thus it is oxygen deficient, similar to the moiré with the triangular dislocation loops. As the cell contains $120 \mathrm{Pt}$ atoms per (111) layer, the $\mathrm{Co}$ and $\mathrm{O}$ densities are 75 and $68 \%$ of the Pt density, respectively. As expected for a structure with line defects, this is less than in the defect-free moiré (81\%).

This structure is reminiscent of the so-called z' phase of $\mathrm{TiO}_{\mathrm{x}} / \mathrm{Pt}(111)(\mathrm{Refs} .39,40)$, even though the $\mathrm{TiO}_{\mathrm{x}} / \operatorname{Pt}(111)$ structure has a smaller unit cell (larger lattice constant in the oxide layer) and the zigzag rows appear bright, not dark in the STM images of $\mathrm{TiO}_{\mathrm{x}} / \mathrm{Pt}(111)$. A recent model for this structure ${ }^{41}$ is similar to ours, but with the $\mathrm{O}$ and metal lattices exchanged $(\mathrm{O}$ forms the upper layer, nevertheless). Thus, in the $\mathrm{TiO}_{\mathrm{x}} / \mathrm{Pt}(111)$ model, the straight line defects are in the $\mathrm{Ti}$, not the $\mathrm{O}$ layer, and the oxygen density exceeds the Ti density.

\section{Multilayer growth}

In Section IIIA, we have discussed the growth and structure of an almost flawless 2D Co oxide layer on the $\operatorname{Pt}(111)$ surface. However the large majority of applications require a film a few nanometers thick exhibiting the electric and magnetic properties characteristic of the $\mathrm{CoO}$ bulk phase. Inspired by Ref. 26, we have repeated the procedure for the moiré several times in the attempt to grow such a thicker layer. Fig. 6(a) shows a large scale STM image measured on a film prepared as follow: $1 \mathrm{ML}$ Co was deposited at RT; it was annealed at $570 \mathrm{~K}$ and it was dosed with $\approx 50 \mathrm{~L} \mathrm{O}_{2}$; then two further cycles of Co deposition $(1 \mathrm{ML})$ and $\mathrm{O}_{2}$ dosing $(50 \mathrm{~L})$ were performed, always at $570 \mathrm{~K}$; finally it was annealed for 10 minutes at $740 \mathrm{~K}$ in $10^{-8}$ mbar $\mathrm{O}_{2}$. We observe large 
$3 \mathrm{D}$ islands, about $5 \mathrm{~nm}$ high, with a $2 \mathrm{D}$ oxide layer in between. The latter is the 2D moiré pattern of a single CoO layer [the STM images of the moiré shown in Fig. 3(a-e) were collected on this sample]. The image also shows many indications of tip instability, especially when scanning over the $3 \mathrm{D}$ islands, which indicates a semiconducting or insulating nature of the islands (cf. scanning tunneling spectroscopy data below). We conclude that this Stranski-Krastanov growth is the thermodynamically stable configuration, and thicker flat oxide films can be obtained only by decreasing the mobility of the atoms at the surface.

We then grew $\mathrm{CoO}$ films following the procedure of Ref. 8, consisting also in cycles of Co deposition $(1 \mathrm{ML})$ and $\mathrm{O}_{2}$ dosing $(50 \mathrm{~L})$, but at lower temperature. The first ML was evaporated at RT, and then the substrate temperature was increased to $450 \mathrm{~K}$ for annealing, oxidizing and the following deposition/oxidation cycles. Fig. 6(b) shows the STM image of the surface after three cycles. The growth is still of Stranski-Krastanov type, but the 3D islands are significantly smaller. They are between 1 and $2 \mathrm{~nm}$ high and have a roughly triangular shape, with the sides oriented like the basis vectors of the Pt surface hexagonal mesh. A surface X-ray diffraction experiment performed on samples growth in the same way has shown that the islands structure fit very well with $\mathrm{CoO}(111)$ rocksalt nanostructures in orientational epitaxy, with bulk interatomic distances. ${ }^{42}$ 

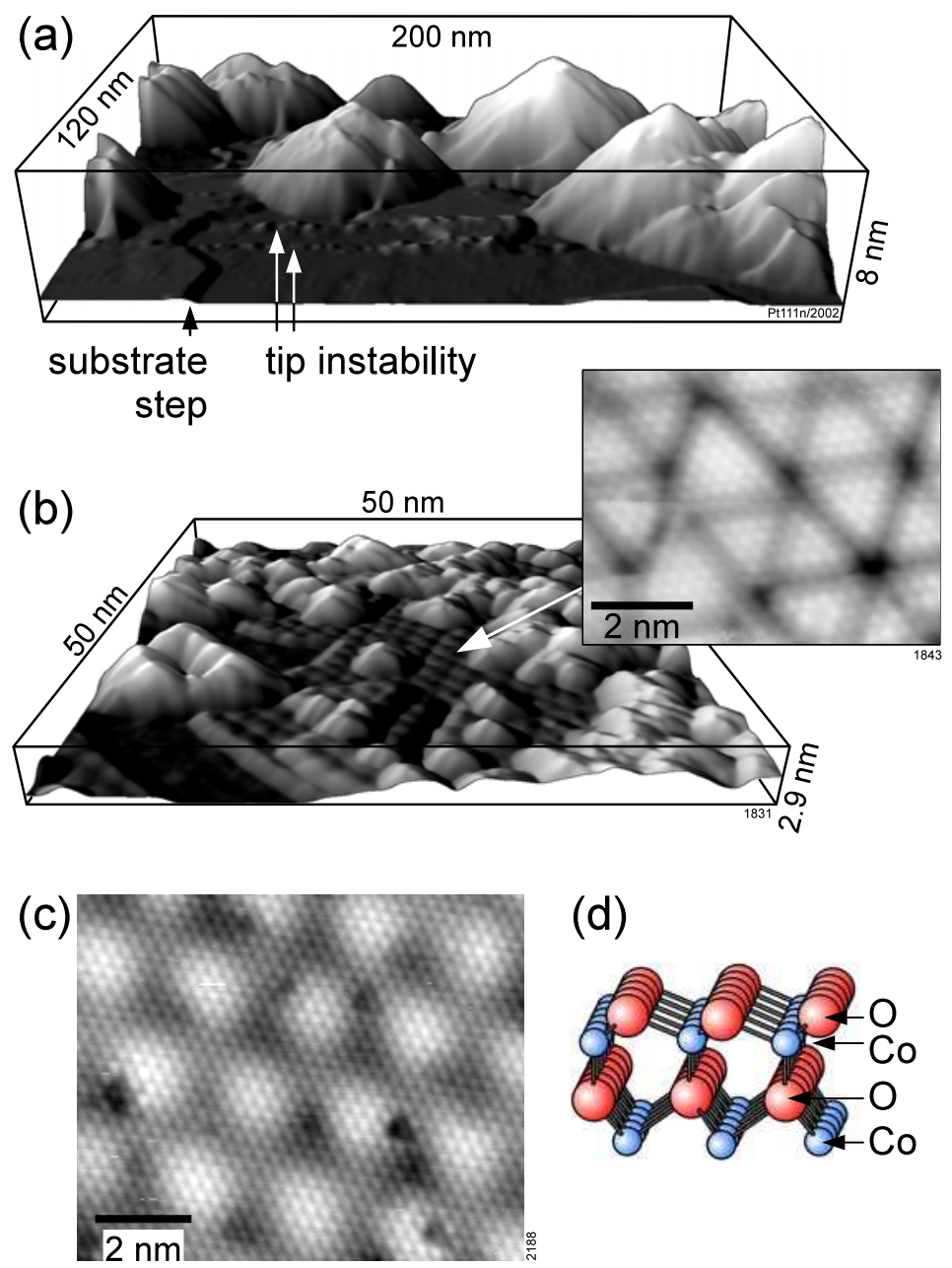

FIG. 6. STM images of thicker films: (a) three cycles of Co deposition (1 ML) and $\mathrm{O}_{2}$ dosing $(50 \mathrm{~L})$ at $570 \mathrm{~K}$, followed by annealing in oxygen at $740 \mathrm{~K}$ at the end $\left(V_{s}=1.2 \mathrm{~V}, I_{t}=0.1 \mathrm{nA}\right) ;(\mathrm{b})$ the same procedure at $T=450 \mathrm{~K}\left(V_{s}=-1 \mathrm{~V}, I_{t}=\right.$ $0.1 \mathrm{nA}$ ). In both cases the first ML is deposited at RT. (c) shows the second-layer moiré structure after depositing $1 \mathrm{ML}$ Co at RT, annealing and dosing $\mathrm{O}_{2}$ at $570 \mathrm{~K}$, then $740 \mathrm{~K}$. (d) Ball model of wurtzite-terminated cobalt oxide.

The second monolayer [inset in Fig. 6(b)] shows a moiré-like structure with many defects; partly reminiscent of the $\mathrm{Cr} / \mathrm{Pt}(111)$ pinwheel $^{43}$ and the $\mathrm{VO}_{\mathrm{x}} / \mathrm{Rh}(111)$ 'wagon-wheel' ${ }^{44}$ structures, though these are clearly different from the current case (pure metal and single oxide layers, respectively). In contrast to the first-layer $\mathrm{CoO} / \mathrm{Pt}(111)$ moiré, the appearance of this second-layer structure does not significantly change with tunneling voltage. We have also obtained patches of a more well-ordered form of this second-monolayer structure by the preparation leading to the perfect 1st-layer moiré; the 2nd-layer structure appears in islands where Co has been expelled due to the increase of the lattice constant when forming the oxide. In this case, the 2 nd layer forms an almost perfect moiré structure, with a supercell size between 2.3 and $2.4 \mathrm{~nm}$ and hardly any rotation with respect to the Pt substrate [Fig. 6(c)]. This means that the lattice constant of the 2-layer structure is approx. 315 pm, larger than that in the 1st-layer moiré (309 pm; $2.7 \mathrm{~nm}$ supercell). As the CoO 
layer forming the 1st-layer moiré has a lattice constant larger than bulk $\mathrm{CoO}$, it would be very unusual to have a 2-layer oxide with the same structure, but a lattice constant deviating even more from the bulk value. We therefore believe that it is a different structure; most likely a wurtziteterminated cobalt oxide as already observed for $\mathrm{CoO}$ on $\operatorname{Ir}(100)$ [Fig. 6(d), Ref. 19]. CoO wurtzite has a lattice constant $a_{\mathrm{w}}=324 \mathrm{pm}$; the actual lattice constant of the two-layer structure will be an average of the rocksalt and the wurtzite ones. As in the $\mathrm{CoO} / \operatorname{Ir}(100)$ case, the reason for the appearance of the wurtzite structure can be explained by the electrostatic energy: The spacing between the $\mathrm{Co}$ and $\mathrm{O}$ planes of a bilayer is $44 \mathrm{pm}$ in the wurtzite structure ${ }^{45}$ or even lower $^{46}$, to be compared to $123 \mathrm{pm}$ in the rocksalt structure. The absence of strong density-of-states effects in STM images of the wurtzite phase, as compared to the 1st-layer moiré, might be related to the absence of a well-developed bandgap in wurtzite $\mathrm{CoO}$, in contrast to the rocksalt structure. ${ }^{46}$

Scanning tunneling spectroscopy $(\mathrm{STS})^{47}$ curves obtained on two different regions of the sample in Fig. 6(b), i.e. on top of the 2-ML islands and on the 3D oxide layer are shown in Fig. 7. To avoid excessive noise in the bandgap, where $I \approx 0$, and thus $\ln I$ is a very high negative number, we do not display the unmodified normalized conductance, $\mathrm{d} \ln I / \mathrm{d} \ln V$ but rather $(\mathrm{d} I / \mathrm{d} V) /(I / V+\mathcal{E})$ with a small $\varepsilon=10 \mathrm{pA} / \mathrm{V}$. The curves show a bandgap in the $3 \mathrm{D}$ islands between -0.4 and $+0.5 \mathrm{~V}$, while the $1^{\text {st }}$-layer (not shown) and $2^{\text {nd }}$-layer moiré structures are still metallic. The gap in bulk CoO is larger, about $3 \mathrm{eV}$ (Ref. 48).

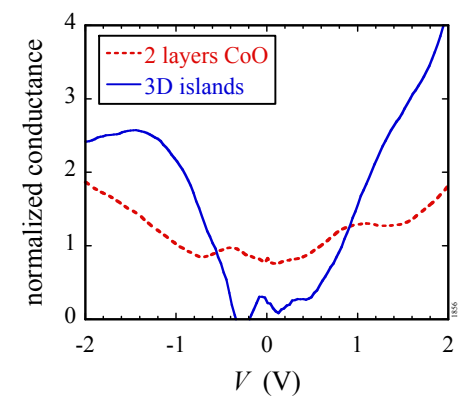

FIG. 7. (Color online) STS measured on the 2-layer oxide and the 3D islands of the preparation shown in Fig. 6(b).

\section{Surface alloy oxides.}

The results discussed in the previous paragraphs highlight the role of the interface interaction in the oxide morphology and show that excess Co above the first layer completion results in StranskiKrastanov growth. Therefore, the use of a surface alloy as Co reservoir looks appealing for growing flat surface oxides. It is well known that annealing a Co ultrathin film deposited on Pt(111) above $670 \mathrm{~K}$ results in a Pt rich surface alloy, which is terminated by a Pt-rich layer. ${ }^{49,50}$ Under oxidizing 
conditions, the Co amount required for growing an oxide layer can migrate to the surface.

We have evaporated $6 \mathrm{ML}$ Co on $\mathrm{Pt}(111)$ at RT and annealed it at $740 \mathrm{~K}$. Finally, $50 \mathrm{~L}$ of $\mathrm{O}_{2}$ were dosed at $640 \mathrm{~K}$. STM measurements [Fig. 8(a)] show an almost flat surface over hundreds of nanometers, with roughly triangular depressions, appearing about $70 \mathrm{pm}$ deep in the surface. Atomically resolved images (inset on the right side of the figure) show that the matrix is the clean metal (probably mostly Pt; we have not achieved chemical contrast in these areas), while the darker regions correspond to the moiré phase (inset on the left). Its appearance at different voltages (not shown) is the same as for the 1-ML-thick oxide films (Fig. 3). STS does not show a bandgap as observed for thicker oxides, and also the lattice constant suggests that it is a single CoO layer: An analysis of the dislocation pattern weakly visible in the images suggests that the alloy substrate has about $272 \mathrm{pm}$ interatomic distance; and the STM images show that the oxide has a lattice constant 1.126 times that of the substrate, i.e., $\approx 306 \mathrm{pm}$ for the oxide lattice, reasonably close to the value of $309 \mathrm{pm}$ found for the oxide after deposition of $1 \mathrm{ML}$ Co.

\section{E. The quasi- $(3 \times 3)$ surface.}

We have also studied oxidation of a surface with a higher Co concentration in the near-surface layers, obtained by annealing at a lower temperature of $640 \mathrm{~K}^{51}$ In this case, 3 Co ML were evaporated at room temperature, annealed at $640 \mathrm{~K}$, and finally dosed with $50 \mathrm{~L} \mathrm{O}_{2}$ at this same temperature [Fig. 8(b)]. Apart from bright subsurface dislocation lines due to the lattice misfit between the Pt substrate and the PtCo alloy, we find a flat homogeneous surface over several tens of nanometers. We also observe three rotational domains of a grating structure with a corrugation of $\approx 10 \mathrm{pm}$ and approx. $3.3 \mathrm{~nm}$ periodicity. High-resolution images (inset in the figure) show that the surface is nanostructured with holes, forming a superstructure with a periodicity close to $(3 \times 3)$, confirmed by LEED; we therefore name it quasi- $(3 \times 3)$ structure. We interpret this phase as the result of the oxidation of a Co rich surface on top of the alloy. The same quasi- $(3 \times 3)$ phase was also observed after deposition of 2 Co ML, which was deposited at RT, annealed first and then dosed with $50 \mathrm{~L} \mathrm{O}_{2}$ at $570 \mathrm{~K}$, and finally dosed with $2 \mathrm{~L}$ at $740 \mathrm{~K}$ [Fig. 9(a)]. A third route towards preparation of this structure is deposition of $7 \mathrm{ML}$ Co onto the 1-ML moire in $10^{-7} \mathrm{mbar}_{2}$ at 740 $\mathrm{K}$; all these methods result in a Pt-Co alloy in the uppermost layers. When grown by the 2nd method ( $2 \mathrm{Co}$ ML annealed at $570 \mathrm{~K}$ and then at $740 \mathrm{~K}$ in oxygen), the quasi- $(3 \times 3)$ superstructure coexists with the moiré phase, which grows preferentially close to steps and with a high density of triangular dislocation loops. 

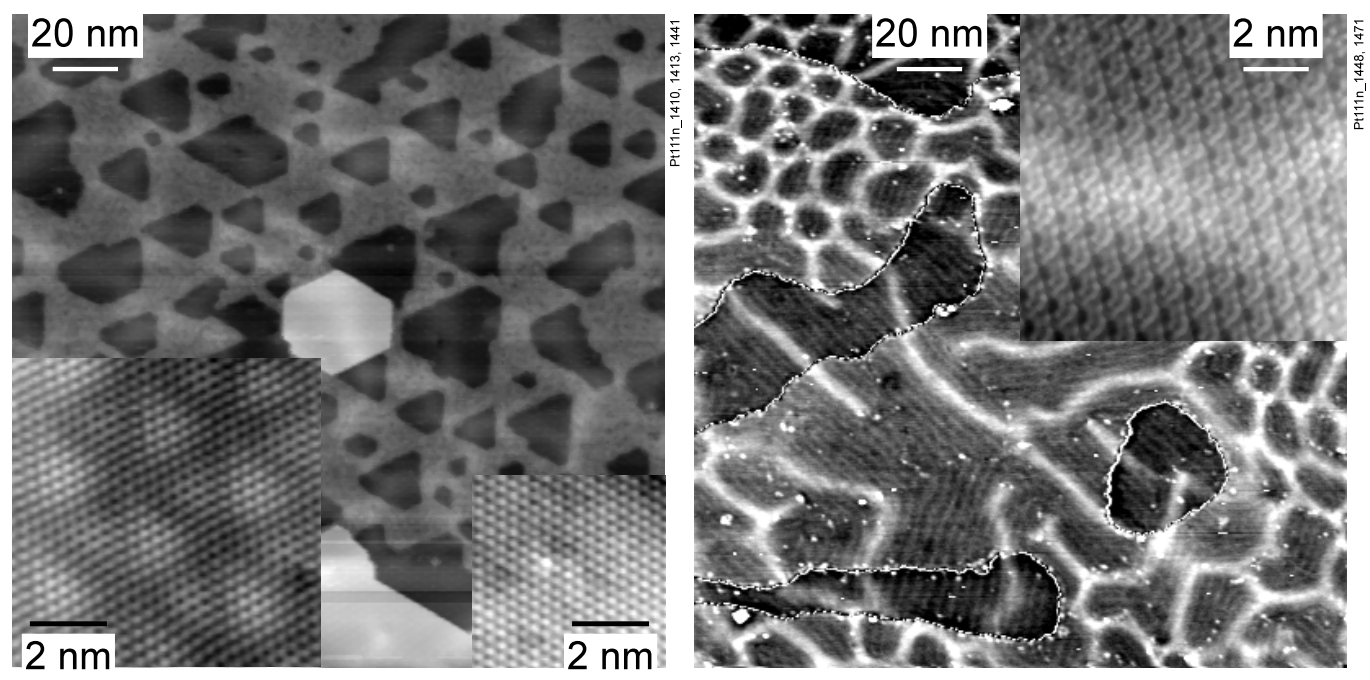

FIG. 8. (a) STM images of $6 \mathrm{ML} \mathrm{Co/Pt(111)} \mathrm{annealed} \mathrm{at} 740 \mathrm{~K}$ and then oxidized at $640 \mathrm{~K}\left(V_{s}=+1 \mathrm{~V}, I_{t}=0.1 \mathrm{nA}\right)$. The insets on the left and on the right side show a zoom in the dark triangles $\left(\mathrm{V}_{\mathrm{s}}=+50 \mathrm{mV}, \mathrm{I}_{\mathrm{t}}=0.1 \mathrm{nA}\right)$ and in the matrix $\left(V_{s}=-1 \mathrm{mV}, I_{t}=2.5 \mathrm{nA}\right)$, respectively. (b) STM images of $3 \mathrm{ML} \mathrm{Co} / \mathrm{Pt}(111)$ annealed and dosed at $640 \mathrm{~K}\left(V_{s}=-1 \mathrm{~V}, I_{t}=\right.$ $0.1 \mathrm{nA}$ ). Image processing has been used for better visibility of low-corrugation features on terraces, causing the noisy appearance of the steps. The inset shows a high-resolution image $\left(\mathrm{V}_{\mathrm{s}}=2 \mathrm{mV}, \mathrm{I}_{\mathrm{t}}=1 \mathrm{nA}\right)$.

A careful analysis of the STM images allows us to determine the exact unit cell of the quasi $(3 \times 3)$ structure with respect to the PtCo alloy. In the following, we will assume that the alloy has the lattice constant of pure Pt; the actual values might be slightly lower due to the smaller alloy lattice constant. The lattice match is exact only in the fcc $\left[\begin{array}{lll}2 & \overline{1} & \overline{1}\end{array}\right]$ direction, with the spacing between the dark spots measured as $1.45+/-0.01 \mathrm{~nm}$ in this direction, which compares very well with $3 \times \sqrt{ } 3 \times d_{P t}=$ $1.442 \mathrm{~nm}$. In the [ $\left[\begin{array}{lll}0 & \overline{1} & 1\end{array}\right]$ direction, the oxide layer is expanded by about $10 \%$ compared to an ideal $(3 \times 3)$ superstructure; the spacing between the dark holes being $0.92+/-0.01 \mathrm{~nm}$, instead of $3 \times d_{P t}=$ $0.833 \mathrm{~nm}$. This is close to the misfit between the $\mathrm{Pt}$ and $\mathrm{CoO}$ lattice constants and is responsible for the $\approx 3.3$-nm periodicity of the grating mentioned above. This periodicity is also seen in atomically resolved images as a weak modulation of contrast [Fig. 9(a)] and allows us to determine the exact unit cell, which is described by the matrix $\left(\begin{array}{cc}13 & 3 \\ 3 & 6\end{array}\right)$. Hence, the unit vectors of the superstructure cell are $\left|a_{S 1}\right|=\sqrt{139} d_{P t}=3.272 \mathrm{~nm}$, which fits the experimental value of $\approx 3.3 \mathrm{~nm}$, and $\left|a_{S 2}\right|=1.442$ nm. This structure can also be described by a centered rectangular cell, $(23 \times 3 \sqrt{3})$-rect. The superstructure is also visible in LEED [Fig. 9(b)]. There, the expansion manifests itself as inwards movement of four of the 6 main spots [hexagon in Fig. 9(b)]; all spots appear as triplets due to the three different rotational domains. 

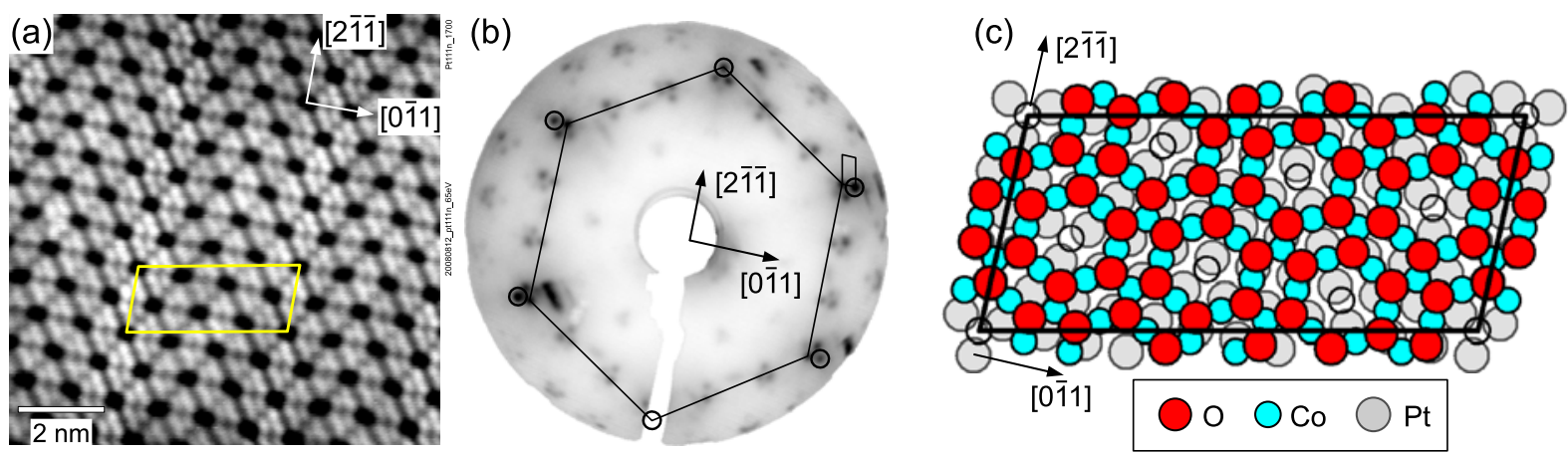

FIG. 9. (Color online) The quasi-(3×3) phase: (a) STM image $\left(V_{s}=-2 \mathrm{mV}, I_{t}=0.1 \mathrm{nA}\right)$. (b) LEED pattern at $65 \mathrm{eV}$ with the substrate spots marked by circles and a large hexagon showing the expansion in the $<0 \quad \overline{1} 1>$ direction. (c) Structure model. The superstructure cell is marked in all frames.

Assuming that our STM shows the oxygen atoms as on all the other Co oxides, we arrive at the structural model shown in Fig. 9(c). This structure is similar to the $(3 \times 3)$ structure observed by Knudsen et al. ${ }^{38}$ for oxidized iron on $\operatorname{Pt}(111)$, but the deviation from the perfect $(3 \times 3)$ superstructure was not reported in that work. The Co layer is almost pseudomorphic to the substrate, but expanded

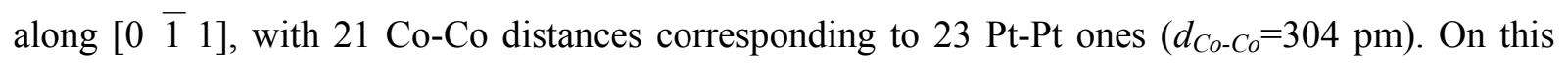
structure, triangles of $3 \mathrm{O}$ atoms have two different orientations; which would correspond to fcc and hcp sites on a perfect $(3 \times 3)$ superstructure. We might think of these triangles as a miniature version of the triangular dislocation loops discussed in section 3B. Due to the expansion along the [ $\left[\begin{array}{lll}0 & \overline{1} & 1\end{array}\right]$ direction, exact fcc and hep stacking is found only at the left and right sides of the unit cell; in the rest of the cell the stacking is between these two extremes. Thus, the unfavorable hcp stacking is not avoided in this structure. The other type of unfavorable stacking, Co on top of $\mathrm{Pt}$, is avoided by keeping commensurability along [2 $\overline{1} \overline{1}]$. In agreement with what has been discussed for the other 1st-layer oxides, the oxygen triangles with perfect fcc stacking show the strongest atomic contrast in STM.

We cannot directly determine whether the Co atoms drawn as open circles in Fig. 9(c), each surrounded by 6 oxygen triangles, are present or not. If present, these atoms would have no oxygen atom binding to them and could also be $\mathrm{Pt}$ (which has a weaker affinity for oxygen than Co). The STM images show a dark hole at these positions, but the depression appears only $\approx 40 \mathrm{pm}$ deep, much less than the geometrical depth of a monolayer-deep hole. We consider it likely that a metal atom at this position would appear as a protrusion in STM, because the first-layer oxide appears darker than a Pt monolayer [Fig. 8(a)]. Also, in a few STM images (not shown) we have observed some 'holes' replaced by protrusions, indicating that an atom can indeed bind there and appear bright. We therefore consider it likely that this atom is indeed missing in the usual quasi- $(3 \times 3)$ superstructure, as already proposed by Knudsen et al. for the $\mathrm{FeO}_{\mathrm{x}} / \mathrm{Pt}(111)$ case. ${ }^{38}$ We should finally 
mention that we have also observed small patches of the $(2 \times 2)$ cobalt oxide phase, similar to that of iron oxide reported by Knudsen et al. (not shown).

\section{Summary and conclusion}

We show that oxidation of Co films deposited on $\operatorname{Pt}(111)$ results in several phases with different morphology depending on the preparation method, on the film thickness and on the annealing temperature before and during oxygen dosing. Deposition of $1 \mathrm{ML}$ of Co and subsequent $\mathrm{O}_{2}$ dosing in the temperature range between $\mathrm{RT}$ and about $470 \mathrm{~K}$ results in a rough surface. This is explained with an excess of $\mathrm{Co}$, which is expelled from the almost pseudomorphic $\mathrm{Co} / \mathrm{Pt}$ (111) layer during the oxide growth. A flat and almost flawless moiré pattern is obtained with oxidation at two increasing temperatures $(570 \mathrm{~K}$ and $740 \mathrm{~K})$ of a $1 \mathrm{ML}$ thick Co deposit. At the final temperature, excess Co can diffuse into bulk. This shows that the moiré is the thermodynamically stable 2D cobalt oxide phase on $\mathrm{Pt}(111)$. AES and the striking analogy to the well-known $\mathrm{FeO} / \mathrm{Pt}(111)$ case allow us to assign this moiré structure to an oxygen-terminated $\mathrm{CoO}(111)$ bilayer. The in-plane distance is slightly expanded (309 pm) compared to a truncated $\mathrm{CoO}(111)$ crystal $(301 \mathrm{pm})$. This is probably associated with an out of plane contraction, which would reduce the surface dipolar energy. Although we did not find evidence for a band gap in STS, the STM images of the moiré structure strongly depend on the tunneling voltage, which is more typical for semiconductors than for a metal. Interestingly, the two-layer moiré structure has an even larger in-plane lattice constant $(315 \mathrm{pm})$. Thus, we believe that it has the wurtzite structure, with the Co of the upper bilayer on top of the $\mathrm{O}$ atoms of the lower bilayer [Fig. 6(d)], similar to $\mathrm{CoO} / \operatorname{Ir}(100){ }^{19}$

Insufficient oxidation of a single Co layer, whether deposited at rather low temperatures or derived from a near-surface PtCo alloy, leads to various substoichiometric structures. In the sequence of decreasing oxygen content, these are the moiré structure with dislocation triangles, the zigzag structure and the quasi- $(3 \times 3)$ structure; the latter one is obtained from near-surface PtCo alloys. With decreasing oxygen content, the Co-Co interatomic distance decreases, but even in the quasi-(3×3) structure with a stoichimetry of $\mathrm{CoO}_{0.75}$, it is still larger than in bulk Pt (277.5 pm), much higher than in bulk Co $(250.7 \mathrm{pm})$. The dislocation triangles and the zigzag structure allow the system to avoid unfavorable stacking sequences of the upper three layers (Pt-Co-O). It is interesting to note that the quasi- $(3 \times 3)$ structure, having the lowest oxygen content of all, does not avoid the unfavorable Pt-Co-O hcp stacking. The zigzag structure is more effective in this respect avoiding both Co-on-top-of-Pt and Pt-Co-O hcp stacking.

Both, the 1st and 2nd-layer moiré structures might find applications as templates for growing metal nanostructures. Applications involving noble metals seem more promising than attempts to 
deposit more reactive transition metals, which would tend react with the oxygen and destroy the moiré pattern. Nevertheless, the similar FeO/Pt(111) moiré has been recently used for growing fairly regularly arranged $\mathrm{Fe}$ and $\mathrm{V}$ clusters; ${ }^{11}$ self-assembling arrays of gold adatoms on this substrate $^{12}$ show much better order, however. To our knowledge, the impact of deviations from the perfect moiré (triangular dislocation loops, zigzag phase) on metal nucleation has not been investigated so far on any similar oxide. As one might expect that the regular defects act as nucleation centers, these surfaces look highly promising as templates.

Thicker films of $\mathrm{CoO}$ on $\mathrm{Pt}(111)$ grow in Stranski-Krastanov mode, which becomes increasingly pronounced with increasing deposition and annealing temperature. Stranski-Krastanov cannot be avoided by cycles of Co deposition and oxidation at elevated temperature. In agreement to the 3-fold symmetry of the 3D islands in these films, surface X-ray diffraction measurements, following such a step-by-step growth at $450 \mathrm{~K}$, show that the island structure agrees very well with the bulk $\mathrm{CoO}(111)$ rocksalt structure, in orientational epitaxy with the substrate. ${ }^{42}$ As expected for $\mathrm{CoO}$, the 3D nanoislands are semiconducting as shown by STS, though with a smaller bandgap than the bulk material. Considering the importance of the interface in exchange bias structures, a careful analysis of the $\mathrm{CoO}$ films like in the present study is clearly required for all work where $\mathrm{CoO}$ films or $\mathrm{CoO}-\mathrm{NiO}$ combinations are used as antiferromagnetic materials for such applications.

We should finally mention that sputter-cleaned STM tips consistently show the oxygen, not the metal atoms as protrusions in all of these structures, where a complete or almost complete closepacked oxygen layer is at the surface (at low coverages, oxygen usually appears as a depression in

$\mathrm{STM}$ ). The same was reported for various $\mathrm{O} / \mathrm{Rh}$ structures, ${ }^{34,52}$ and we consider it likely that this may be a general rule for STM images of oxygen on the late transition metals.

\section{Acknowledgement}

This work was supported by the Austrian Fonds zur Förderung der wissenschaftlichen Forschung. 


\section{References}

*Corresponding author; Permanent address: Institut Néel, UPR 2940 - CNRS, 25 av. des Martyrs, BP166, 38042 Grenoble, France; Email: Maurizio.De-Santis@grenoble.CNRS.Fr

${ }^{1}$ E. Lundgren, A. Mikkelsen, J. N. Andersen, G. Kresse, M. Schmid and P. Varga, J. Phys.: Condens. Matter 18, R481 (2006).

${ }^{2}$ U. Diebold, S-C. Li and M. Schmid, Annu. Rev. Phys. Chem. 61, 129 (2010).

${ }^{3}$ A. E. Berkowitz, K. Takano, J. Mag. Mag. Mater. 200, 552 (1999).

${ }^{4}$ W. H. Meiklejohn and C. P. Bean, Phys. Rev. 102, 1413 (1956).

${ }^{5}$ V. Skumryev, S. Stoyanov, Y. Zhang, G.. Hadjipanayis, D. Givord and J. Nogués, Nature 423, 850 (2003).

${ }^{6}$ M. Gruyters and D. Riegel, Phys. Rev. B 63, 052401 (2000).

${ }^{7}$ J. A. Borchers, M. J. Carey, R. W. Erwin, C. F. Majkrzan and A. E. Berkowitz, Phys. Rev. Lett. 70, 1878 (1993).

${ }^{8}$ J. M. Tonnerre, M. De Santis, S. Grenier, H. C. N. Tolentino, V. Langlais, E. Bontempi, M. Garcia-Fernandez and U. Staub, Phys. Rev. Lett. 100, 157202 (2008).

${ }^{9}$ A. Buchsbaum, M. De Santis, H. C. N. Tolentino, M. Schmid, and P. Varga, Phys. Rev. B 81, 115420 (2010) and Refs. therein.

${ }^{10}$ S. Degen, C. Becker, and K. Wandelt, Faraday Discuss. 125, 343-356 (2004).

${ }^{11}$ N. Berdunov, G. Mariotto, K. Balakrishnan, S. Murphy and I. V. Shvets, Surf. Sci. 600, L287 (2006).

${ }^{12}$ L. Giordano, G. Pacchioni, J. Goniakowski, N. Nilius, E. D. L. Rienks and H.-J. Freund, Phys. Rev. Lett. 101, 026102 (2008).

${ }^{13}$ E. Cavaliere, I. Kholmanov, L. Gavioli, F. Sedona, S. Agnoli, G. Granozzi, G. Barcaro, and A. Fortunelli, Phys. Chem. Chem. Phys. 11, 11305 (2009).

${ }^{14}$ S. Hayazaki, T. Matsui, H. Zhang, W. Chen, A. Wee, and J. Yuhara, Surf. Sci. 602, 2025-2028 (2008).

${ }^{15}$ J. W. Tasker, J. Phys. C, 12, 4977 (1979).

${ }^{16}$ J. Goniakowski, F. Finocchi, and C. Noguera, Rep. Prog. Phys. 71, 016501 (2008).

${ }^{17}$ C. Noguera, J. Phys.: Condens. Matter 12, R367 (2000).

${ }^{18}$ D. Wolf, Phys. Rev. Lett. 68, 3315 (1992).

${ }^{19}$ W. Meyer, D. Hock, K. Biedermann, M. Gubo, S. Müller, L. Hammer, and K. Heinz, Phys. Rev. Lett. 101, 016103 (2008).

${ }^{20}$ O. Dulub, U. Diebold, and G. Kresse, Phys. Rev. Lett. 90, 016102 (2003).

${ }^{21}$ W. Hebenstreit, M. Schmid, J. Redinger, R. Podloucky, and P. Varga, Phys. Rev. Lett. 85, 5376 (2000).

${ }^{22}$ C. Noguera and J. Goniakowski, J. Phys.: Condens. Matter 20, 264003 (2008). 
${ }^{23}$ M. Hassel and H.-J. Freund, Surf. Sci. 325, 163-168 (1995).

${ }^{24}$ W. Meyer, K. Biedermann, M. Gubo, L. Hammer, and K. Heinz, Phys. Rev. B 79, 121403(R) (2009).

${ }^{25}$ L. Gragnaniello, S. Agnoli, G. Parteder, A. Barolo, F. Bonbino, F. Allegretti, S. Surnev, G. Granozzi and F. P. Netzer, Surf. Sci. 604, 2002 (2010).

${ }^{26}$ S. Entani, M. Kiguchi and K. Saiki, Surf. Sci. 566-568, 165 (2004).

${ }^{27}$ C. Nagl, O. Haller, E. Platzgummer, M. Schmid and P. Varga, Surf. Sci. 321, 237 (1994).

${ }^{28}$ E. Lundgren, B. Stanka, M. Schmid and P. Varga, Phys. Rev. B 62, 2843 (2000).

${ }^{29}$ E. Lundgren, B. Stanka, W. Koprolin, M. Schmid and P. Varga, Surf. Sci. 423, 357 (1999).

${ }^{30}$ H. C. Galloway, J. J. Benitez and M. Salmeron, Surf. Sci. 298, 127 (1993).

${ }^{31}$ H. C. Galloway, P. Sautet and M. Salmeron, Phys. Rev. B 54, R11145 (1996).

${ }^{32}$ M. Ritter, W. Ranke and W. Weiss, Phys. Rev. B 57, 7240 (1998).

${ }^{33}$ Y. J. Kim, C. Westphal, R. X. Ynzunza, H. C. Galloway, M. Salmeron, M. A. Van Hove and C. S. Fadley, Phys. Rev. B 55, R13448 (1997).

${ }^{34}$ J. Gustafson, A. Mikkelsen, M. Borg, E. Lundgren, L. Köhler, G. Kresse, M. Schmid, P. Varga, J. Yuhara, X. Torrelles, C. Quirós, and J. N. Andersen, Phys. Rev. Lett. 92, 126102 (2004).

${ }^{35}$ Scientific Group Thermodata Europe (SGTE), P. Franke, D. Neuschütz, Landolt-Börnstein - Numerical Data and Functional Relationships in Science and Technology, Group IV, Volume 19B (Springer, Berlin 2004).

${ }^{36}$ L. Giordano, G. Pacchioni, J. Goniakowski, N. Nilius, E. D. L. Rienks, and H. Freund, Phys. Rev. B 76, 075416 (2007).

${ }^{37}$ L. R. Merte, J. Knudsen, L. C. Grabow, R. T. Vang, E. Lægsgaard, M. Mavrikakis and F. Besenbacher, Surf. Sci. 603, L15 (2009).

${ }^{38}$ J. Knudsen, L. R. Merte, L. C. Grabow, F. M. Eichhorn, S. Porsgaard, H. Zeuthen, R. T. Vang, E. Lægsgaard, M. Mavrikakis, and F. Besenbacher, Surf. Sci. 604, 11-20 (2010).

${ }^{39}$ O. Dulub, W. Hebenstreit, and U. Diebold, Phys. Rev. Lett. 84, 3646-3649 (2000).

${ }^{40}$ D. R. Jennison, O. Dulub, W. Hebenstreit, and U. Diebold, Surf. Sci. 492, L677-L687 (2001).

${ }^{41}$ F. Sedona, G. Granozzi, G. Barcaro and A. Fortunelli, Phys. Rev. B 77, 115417 (2008).

${ }^{42}$ M. De Santis, H. C. N. Tolentino, J. M. Tonnerre, A. Bailly, and M.-C. Saint Lager, ECSCD 9 (9th European Conference on Surface Crystallography and Dynamics), Vienna 2-5 Sept. 2007.

${ }^{43}$ L. Zhang, J. van Ek, and U. Diebold, Phys. Rev. B 57, R4285 (1998); Phys. Rev. B 59, 5837 (1999).

${ }^{4}$ [1] J. Schoiswohl, S. Surnev, M. Sock, S. Eck, M. G. Ramsey, F. P. Netzer, and G. Kresse, Phys. Rev. B 71, 165437 (2005).

${ }^{45}$ A. S. Risbud, L. P. Snedeker, M. M. Elcombe, A. K. Cheetham and R. Seshadri, Chem. Mater. 17, 834 (2005). 
${ }^{46}$ T. Archer, R. Hanafin, and S. Sanvito, Phys. Rev. B 78, 014431 (2008).

${ }^{47}$ R. M. Tromp, J. Phys.:Condens. Matter 1, 10211 (1989).

${ }^{48}$ V. I. Anisimov, M. A. Korotin and E. Z. Kurmaev, J. Phys.: Condens. Matter 2, 3973 (1990).

${ }^{49}$ M. C. Saint-Lager, R. Baudoing-Savois, M. De Santis, P. Dolle, Y. Gauthier, Surf. Sci. 418, 485 (1998).

${ }^{50}$ Y. Gauthier, M. Schmid, S. Padovani, E. Lundgren, V. Buš, G. Kresse, J. Redinger, and P. Varga, Phys. Rev. Lett. 87, 036103 (2001).

${ }^{51}$ R. Baudoing-Savois, P. Dolle, Y. Gauthier, M. C. Saint-lager, M. De Santis and V. Jahns, J. Phys.: Condens. Matter 11, 8355 (1999).

${ }^{52}$ L. Köhler, G. Kresse, M. Schmid, E. Lundgren, J. Gustafson, A. Mikkelsen, M. Borg, J. Yuhara, J. N. Andersen, M. Marsman, and P. Varga, Phys. Rev. Lett. 93, 266103 (2004). 